EXTENDED REPORT

\title{
Ophthalmological manifestations in segmental neurofibromatosis type 1
}

\author{
M Ruggieri, P Pavone, A Polizzi, M Di Pietro, A Scuderi, A Gabriele, A Spalice, P lannetti
}

Br J Ophthalmol 2004;88:1429-1433. doi: 10.1136/bjo.2004.043802

See end of article for authors' affiliations

Correspondence to: Martino Ruggieri, $\mathrm{MD}, \mathrm{PhD}$, Institute of Neurological Science, National Research Council (CNR), Viale R Margherita, 6, 95125-Catania, Italy; m.ruggieri@ism.omr.it

Accepted for publication 27 April 2004

\begin{abstract}
Aims: To study the ophthalmological manifestations in individuals with the typical features of neurofibromatosis type 1 (NF1) circumscribed to one or more body segments, usually referred to as segmental NF1.

Methods: Visual acuity and colour tests, visual field examination, slit lamp biomicroscopy of the anterior segment, and a detailed examination of the retina by indirect ophthalmoscopy were performed at diagnosis and follow up in 72 consecutive subjects (29 males, 43 females; aged 1-64 years; mean age 14.6 years) seen at the university departments of paediatrics in Catania and Rome, Italy, during years 1990-2003, who had in restricted body areas: (1) typical pigmentary manifestations of NF1 (café au lait spots and freckling) only ( $n=48)$; (2) NF1 pigmentary manifestations and neurofibromas alone $(n=2) ;(3)$ neurofibromas only ( $n=15)$; and (4) plexiform neurofibromas only $(n=7)$.

Results: None of the 72 patients had Lisch nodules in the iris irrespective of age at eye examination or hypertelorism (a "minor" NF1 feature) and none developed typical associated ophthalmological NF1 complications. An additional child had an isolated optic pathways glioma (OPG), which behaved both biologically and radiographically as an NF1 associated OPG.

Conclusions: This represents the first systematic study reporting on eye involvement in the largest series of individuals at different ages having segmental NF1. As one of the postulated mechanisms to explain segmental NF1 is somatic mosaicism for the NF1 gene (so far demonstrated only in two patients) the present findings could be explained either by the fact that the eye is too far from the mutated area with NF1 lesions in most cases or by the NF1 (or other "predisposing" or "cooperating") gene mutation restricted to too few cellular clones or to tissues embryologically different from the eye.
\end{abstract}

t is increasingly recognised that the clinical features of many genetic conditions may sometimes become manifest in a mosaic (segmental) form, involving the body in a linear, patchy or otherwise circumscribed arrangement or in localised regions or organs. ${ }^{1-4}$

In the most common form of neurofibromatosis, neurofibromatosis type 1 (NF1), patients with the typical disease features limited to one or more body segments are usually referred to as having segmental (localised) NFl as opposed to the full blown (generalised) phenotype. ${ }^{56}$ Likewise, patients with unilateral features of neurofibromatosis type 2 (NF2) (for example, unilateral vestibular schwannoma accompanied by other ipsilateral tumours) have been reported as having segmental NF2. ${ }^{78}$

Segmental NFl has been widely reported with increasing frequency ${ }^{6-16}$ and is probably underdiagnosed because the clinical picture, as a result of limited signs or symptoms in most of the affected individuals, can be neglected by the patient himself and passes unnoticed by the physician.

From a genetic viewpoint, the so far demonstrated mechanism to explain the localised manifestations of the neurofibromatoses is somatic mosaicism for the NF1 ${ }^{17}{ }^{18}$ or NF2 genes. ${ }^{719}$ Putatively, the clinical presentation depends on the timing of the mutational event in development. When the mutation occurs very early-before tissue differentiationthe clinical phenotype would be generalised; the later a mutation occurs in development the more the phenotype will be confined to a single region or organ. ${ }^{6} 1420$

In order to better define the clinical phenotype of individuals having localised manifestations of NFI and to determine if the eye or limited regions of the eye are involved we studied the ophthalmological manifestations in a cohort of 72 consecutive individuals with segmental NFl ascertained through the neurofibromatoses clinics in Catania and Rome, Italy followed up from 1990 to 2003.

\section{SUBJECTS AND METHODS}

The neurofibromatoses clinics at the departments of paediatrics of the universities of Catania (southern Italy) and Rome (central Italy) were established in 1990 and 1998, respectively, as consultation services primarily to assist in the diagnosis and management of the different forms of neurofibromatosis in children. Children (and also adults), have been referred to the clinic by paediatricians and adult physicians, geneticists, dermatologists, ophthalmologists, neurologists, general practitioners, and through the local and national neurofibromatosis associations because of: (1) a form of neurofibromatosis suspected as a result of presence of pigmentary lesions, cutaneous or subcutaneous nodules, or specific problems related to neurofibromatosis; (2) a previous diagnosis of NF1, NF2 or other forms of neurofibromatosis; (3) a first degree relative affected by neurofibromatosis. The clinics cater for families with neurofibromatosis primarily from the eastern provinces of Sicily (population of approximately 2.6 million inhabitants out of the six million inhabitants of the region Sicily) and the region of Rome (populations of approximately 2.9 million inhabitants), with additional referrals from other Italian regions.

From January 1990 to December 2003, 730 individuals from 412 families were evaluated at these clinics, of whom 600 had been referred and fully assessed for specific concerns regarding a suspect NF1. Overall, 388 out of the 600 had NF 1 according to the NIH criteria ${ }^{21}$ revised $^{15}$ (218 males, 170

Abbreviations: NF1, neurofibromatosis type 1; OPG, optic pathways glioma 
Table 1 Clinical features and inclusion criteria for the study

\begin{tabular}{ll}
\hline Inclusion criteria & No of cases \\
\hline Café au lait spots and freckling only & 48 \\
+ hyperpigmented background in involved area) & \\
Neurofibromas only & 15 \\
$\quad$ Chest region (skin) & $5 / 15$ \\
Chest region (major thoraci nerves) & $3 / 15$ \\
Abdominal region (skin) & $4 / 15$ \\
Limbs(skin + major nerves) & $3 / 15$ \\
Café au lait spots and freckling + neurofibromas & $2 *$ \\
(+ hyperpigmented background in involved area) & \\
Plexiform neurofibromas only & 7 \\
$\quad$ Scalp temporal/occipital region & $3 / 7$ \\
Abdominal region & $2 / 7$ \\
Pelvic region & $2 / 7$ \\
\hline *1 woman also had an NF1 complication (that is, plexiform \\
neurofibroma) (see fig 1A).
\end{tabular}

females; age ranged from 9 months to 77 years, with an average age of 23.2 years); 72 out of the 600 had typical NF1 manifestations confined to a restricted body area (29 males, 43 females; aged 1-64 years; mean age 14.6 years) (see table 1). These were screened at first referral, including all their available first degree relatives, by skin, Wood's lamp, full ophthalmological evaluation, and general and neurological examination. Diagnosis of segmental NFI was made according to the criteria reported elsewhere ${ }^{6}{ }^{14}$ (see also listed features under Results and table 1). Patients referred and/or seen or already being followed up before 1990 were enrolled in the clinics from January 1990.

The further approach of (generalised and segmental) NF1 patients depended on the physician's findings and on patient's acceptance according to the protocol for NFl as reported elsewhere. ${ }^{6} 14152223$

Individuals with segmental NFl had a general medical review, including general and neurological examination and blood pressure measurement, on a yearly basis to monitor the NF1 lesions evolution and disease complications. ${ }^{6}{ }^{1423}$

The ophthalmological protocol included orthoptic evaluation, visual acuity measure, slit lamp biomicroscopy of the anterior segment, a detailed examination of the retina, by means of indirect ophthalmoscopy, and a Goldmann three mirror contact lens, and an applanation tonometry in older children (that is, $>6$ years of age). In younger (that is, $<6$ years of age) or less cooperative children a colour test with Ishihara tables was performed. In older (that is, $>6$ years of age) or more cooperative children a Farnsworth 100 colour test and a computerised visual field evaluation with Humphrey perimeter were performed. The full protocol was performed at diagnosis and at intervals of 1 year. Before being enrolled in the study formal consent was obtained by parents or legal tutors of children and/or by patients themselves.

The following features were specifically searched for at clinical and/or ophthalmological examination: congenital small orbit, plexiform neurofibromas of the orbit, hypertelorism, eyelid and/or periorbital café au lait spots or freckling, eyelid cutaneous, nodular or plexiform neurofibromas, congenital ptosis, exophthalmos or buphthalmos, prominent corneal nerves, Lisch nodules in the iris, congenital or acquired glaucoma, choroidal hamartomas, dilated vessels and any retinal anomaly suggestive of optic nerve pathology.

\section{DNA analysis}

DNA from all the segmental NFl subjects was extracted from peripheral blood leucocytes. Genomic DNA was isolated from total blood samples using standard protocols. For polymerase chain reaction amplification of the 60 exons of the NFl gene published primers were used and analysed by means of DHPLC analysis and DNA sequencing by AG.

\section{RESULTS}

The localised NF1 manifestations in our study group fell into three main clinical subgroups: (1) pigmentation anomalies (café au lait spots and freckling) with (fig 1A) or without neurofibromas $(\mathrm{n}=50)$; (2) (dermal and/or nodular) neurofibromas only $(\mathrm{n}=15)$ (fig $1 \mathrm{~B})$; and (3) plexiform neurofibromas only $(n=7)$.

Clinical and ophthalmological examination of the probands revealed no NFl pigmentary lesions (café au lait spots and/or freckling) or neurofibromas in the periorbital region or the eyelids. In the 15 patients who had neurofibromas only these were restricted to specific body areas (see table 1). Seven patients had plexiform neurofibromas as their only NF1 manifestations localised either under the scalp of the temporal or occipital regions $(\mathrm{n}=3)$ sparing the skull bone including the orbit as revealed by computed tomography (CT) and magnetic resonance imaging (MRI) of the head or the
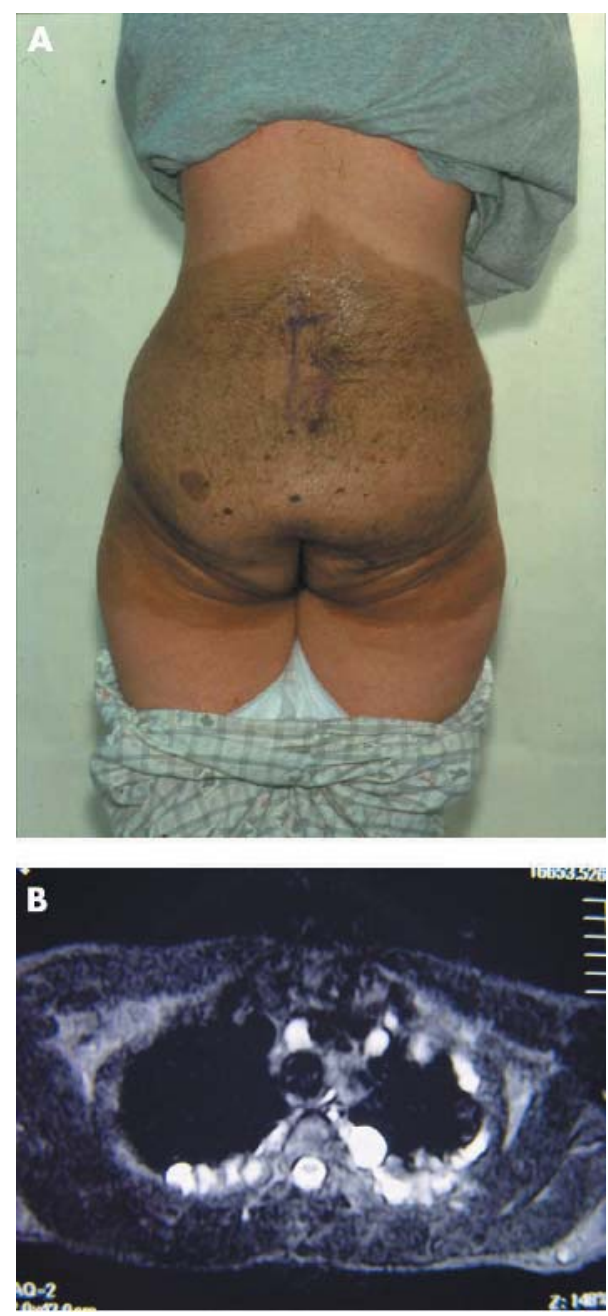

Figure 1 (A) An 18 year old girl with localised manifestations of NF1 shows a quadrant of her body including the lower back, the buttocks and the upper region of the thighs with a darker (hyperpigmented) background and within multiple café au lait spots, freckling, cutaneous neurofibromas, and a large underlying plexiform neurofibroma. No NF1 lesions (or complications) are present in other body regions. Note the sharp cut off in the areas involved with the NFI lesions. (B) Axial CT scan of the chest at the level of the mid-thoracic vertebrae shows a localised streak of nodular neurofibromas in this otherwise healthy 34 year old man. 


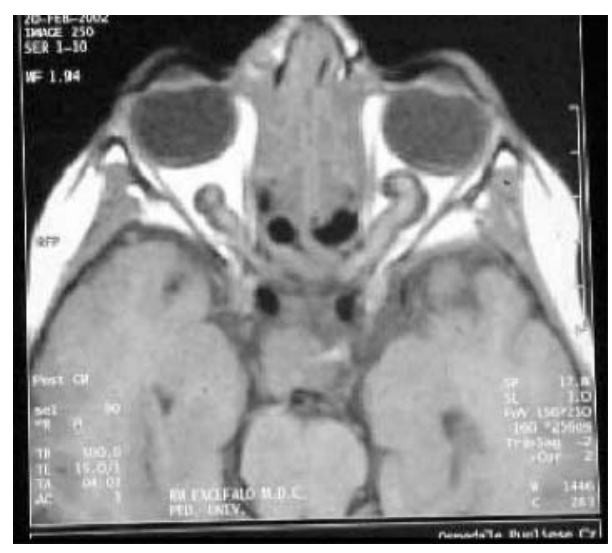

Figure 2 Brain axial $\mathrm{Tl}$ weighted (TR, 450; $\mathrm{TE}, 40$ ) magnetic resonance images shows distortion of the normal architecture and enlargement of both optic nerves and the chiasm with enlarged perioptic spaces in this 9 year old boy who had no other NF1 stigmata.

abdominal $(n=2)$ and pelvic regions $(n=2)$. We did not observe ptosis, exophthalmos, or buphthalmos at any stage of our diagnostic examination and at follow up. Lisch nodules were absent in probands and their relatives. Five parents (two fathers, three mothers) had dark brown naevi localised in the iris.

Funduscopy revealed no anomalies in the probands examined. Notably, out of the 72 patients 15 children (aged 3-11 years, average 7 years 4 months) and five adults (aged 35 and 67 years) with localised manifestations of NF1 were initially suspected-elsewhere in Italy_as having full blown NFl and were enrolled in the full NFl screening protocol used before 1995 in Italy which included, besides clinical and ophthalmological examination, heart and abdominal ultrasound, electroencephalographic examination (EEG), and brain MRI. These investigations all gave normal results. Three further children in this series after being diagnosed as having segmental NFl had either episodes of waxing and waning vertigo or acute headache and were admitted to wards where a brain MRI scan was performed yielding normal results.

A 9 year old child was referred to the department in Catania at age 4 for progressive visual acuity deficits. Upon general examination at first referral he had no pigmentary or nodular lesions and his general examination was normal. Weight, length and head circumference were at the 50th percentile. Neurological examination revealed an alert boy of normal intelligence. Visual acuity was 6/10 in the right eye and 8/10 in the left eye. The Farnsworth 100 colour test and the computerised visual field evaluation with Humphrey perimeter revealed a yellow-blue deficit in both eyes. Funduscopy showed enlarged retinal vessels with a grade 1 papilloedema. Tl weighted images at MRI of the brain showed distortion of the normal architecture and enlargement of both optic nerves (fig 2) and the chiasm; enlarged perioptic spaces with signal intensity identical to cerebrospinal fluid which did not enhance after gadolinium administration. After a 5 year follow up the visual acuity ameliorated and the MRI lesions remained stable with mild involution at his last MRI control (at age 9 years).

No NFl gene deletions, mutations, or microlesions have been detected so far.

\section{DISCUSSION}

The calculated prevalence of segmental NFl in the general population is one in 36000-40000 individuals, or $0.0027 \% .^{6}{ }^{1014}$ Clinically, affected patients manifest their
NFl features in a restricted area varying from one segment to one half of the body or more than one involved segment of the body on both sides of the midline either in a symmetrical or asymmetrical arrangement. ${ }^{6}{ }^{14}$ What is important to understand is that segmental NFl should not be regarded as a separate form of neurofibromatosis but as a localised phenotype of NFl..$^{14} 171824$

Age at presentation of NFl lesions in this series varied according to the presence of pigmentation anomalies only (birth to 2 years of age) or neurofibromas alone (from around puberty to young adulthood). Affected individuals manifested their NFl features in exactly the same way as when they occur in the full blown disease. Despite that, none of the patients in this study group had typical NFl ophthalmological manifestations, including eye complications, regardless of age at examination. It was of interest that the age spanning of this series included the ages most at risk for manifesting the typical NFl ophthalmological features (for example, Lisch nodules) or NFl ophthalmological complications (for example, orbital or intracranial plexiform neurofibromas associated or not with dysplasia of greater wing of sphenoid (1-2 years of age) and optic pathway gliomas (4-9 years of age)). ${ }^{15}{ }^{16}$ Despite this age span and the relatively long follow up in this group and the full screening protocol by means of eye ultrasound and head MRI performed in some children in this group, we could not find any typical NFl ophthalmological complications. Furthermore, most of these patients were at the age of having already developed the major pigmentary lesions of the disease $^{6151623}$ and therefore the lack of café au lait spots and/ or freckling in (restricted areas of) the eyelid and the orbit were also significant.

Lisch nodules were not encountered in the present series regardless of age at eye examination. Lisch nodules have been reported in isolated cases-when the eye was specifically searched for-in previous segmental NFl series and were not always unilateral or ipsilateral to the side of NFl manifestations (reviewed in Huson and Ruggieri ${ }^{6}$ and Ruggieri and Huson $\left.^{14}\right) .{ }^{25}$ Although it is difficult to find a reasonable explanation for these phenomena it can be postulated that this probably occurs because the eye is too far from the mutated area with NFl lesions in most cases or because the NFl (or other "predisposing" or "cooperating" gene) mutation affects tissues or clones different from the eye. Notably, however, patients with manifestations of NFl restricted to the face only reported in previous studies ${ }^{6}{ }^{14}$ (for example, patients having either plexiform neurofibromas or cutaneous and/or nodular neurofibromas of the orbital region or the face as their only NFl manifestations) had no Lisch nodules regardless of age at eye examination. ${ }^{6}{ }^{14}$ There are also two families (Tenconi R, personal communication, 1996 and Riccardi and Lewis ${ }^{25}$ ) with parents having unilateral or bilateral Lisch nodules (that is, only one major diagnostic criterion for NF1) whose sons had generalised NF1. One may question the specificity of iris nodules in NFl as these may (even though rarely) occur in individuals who do not have neurofibromatosis (or at least do not have additional evidence of neurofibromatosis). ${ }^{26}{ }^{27}$ However, though not pathognomonic for neurofibromatosis, iris nodules occur predominantly in individuals with NF1 $(90-100 \%$ of adults with NF1). Therefore, it might be assumed ${ }^{6}{ }^{14}$ that individuals with isolated iris nodules and offspring with NFl (or people with localised features of NFl and Lisch nodules) may have NF1 (or other "predisposing" or "cooperating") gene mutations limited to restricted body areas or specific organs or parts of organs (including, or not, the gonads).

Previous cases of typical NF1 ophthalmological complications in the setting of segmental NFl have been reported in the literature-optic pathway gliomas or orbital plexiform 
neurofibromas (reviewed in Huson and Ruggieri ${ }^{6}$ and Ruggieri and Huson ${ }^{14}$ ). Segmental NFl cases with plexiform neurofibromas of the orbit or the eyelid as their only NFI manifestations have been also reported. ${ }^{6}{ }^{14}$ Whatever figures we take for prevalence of NFl complications in segmental $\mathrm{NF}$ - -including eye involvement-this is certainly much lower (around $7 \%$ in the literature) ${ }^{6}{ }^{14}$ than in the generalised disease. A possible explanation for this phenomenon could be that the NFl gene mutation is restricted to too few cellular clones in most of these patients. ${ }^{17-20}$

In this study one additional child had an isolated optic pathway tumour, which behaved both biologically and radiographically as a typical NF1 associated tumour. Other authors ${ }^{28}$ have recorded similar findings. Even though there has been a considerable debate on whether the clinical and radiological manifestations and natural history of OPGs associated with NFl differ from those of sporadic tumours there are some distinguishing characteristics of NFl OPGs compared to non-OPGs ${ }^{15} 162627$ : (1) initial signs in NF1 OPGs are usually proptosis, precocious puberty, and decreased visual acuity (the latter as in the present case) $v$ increased intracranial pressure/hydrocephalus, nystagmus, and strabismus in non-NFl OPGs; (2) in NFl OPGs the tumour location is common in the intraorbital optic nerve (as in the present case), multicentric, and usually chiasmatic $v$ a rarer location in the intraorbital optic nerve, a unicentric origin usually confined to the optic tract and/or chiasmatic in the non-NFl OPGs; and (3) tumour growth during follow up is uncommon in NFl associated OPGs (like in this child) $v$ a common (and often rapid) growth during follow up in non-NFl OPGs. Thus, even though we did not include this child in the series of segmental NFl patients we reported it in the study as one might theoretically postulate that the isolated OPG (behaving as the typical NFl tumour) could be a localised form of NF1. ${ }^{6}{ }^{14}$ Other authors (RJ Packer, personal communication, November 2003 and Listernick et $a^{28}$ ) have drawn similar conclusions. Notably, cases of isolated long bone dysplasia behaving clinically and radiographically as a typical NF1 associated dysplasia have been reported in association with localised pigmentary features of NF $1 . .^{14} 28$

What is important to understand is that all the clinical and molecular data in this study seem to be compatible with a pathogenic mechanism different from the most commonly postulated explanation for segmental NFl: mosaicism for a NFl gene mutation. ${ }^{6141718}$ We could find no NFl gene mutation in blood DNA in the 72 cases analysed and no ocular NFl abnormalities were seen. Many laboratories have analysed peripheral blood samples and biopsy specimens taken from affected and unaffected skin areas in a large number of cases with segmental NF1 so far ${ }^{14} 29$ and only two reports have shown NFl gene mutations in a mosaic pattern. ${ }^{17}{ }^{18}$ By taking together these data we might hypothesise that segmental NFl could be a heterogeneous condition in which only in very exceptional cases mosaicism for a NFl gene mutation has been found. Thus, the mechanism in the majority of cases might be different. Similar findings have been shown in another localised form (likely a third major form $)^{30}{ }^{31}$ of neurofibromatosis-multiple schwannomatosis (a recently recognised disorder defined as the occurrence of multiple pathologically proved schwannomas without vestibular tumours diagnostic of NF2): only in a minority of cases does multiple schwannomatosis result from mosaicism of a NF2 gene mutation; in the majority of cases the primary genetic defect is linked to a region on chromosome 22 different from NF2, that predisposes or cooperates with somatic (acquired) mutations in the NF2 gene in Schwann cells. 3233

From a genetic counselling viewpoint is of interest that there are a handful of familial cases in the literature where parents with localised manifestations of NFl had children either with full blown ${ }^{561434-37}$ or with localised disease. ${ }^{35}{ }^{38} \mathrm{At}$ present there are no means to exclude, in a given individual with localised manifestations of NFl, involvement of both somatic and gonadal tissue ${ }^{39}$ and, therefore, the exact level of risk is not definable with current knowledge. Studies of pigmentary anomalies in chimeric mice suggest the degree of gonadal involvement might be proportional to the area of skin involved. ${ }^{6}{ }^{14}{ }^{40}$ However, even though the segment of the body affected is distant from the gonads, there can be gonadal involvement with the chance of transmitting the full blown or segmental disease to offspring. Therefore, the presence or lack of Lisch nodules in the setting of segmental NFl (and we would add in any case) ${ }^{27} 28$ should not be considered a predictor for transmission of NFl to offspring.

In conclusion, even though ocular manifestations of NFl, including Lisch nodules and NF1 ophthalmological complications, have been reported in previous isolated cases of segmental NFl we could not confirm these findings in the present systematic study. Whatever figures we take, this means that eye involvement is much less frequent than expected in patients with generalised disease and, therefore, close ophthalmological monitoring in the setting of segmental NFl could be unnecessary. Furthermore, as the risk of typical NFl eye complications in segmental NFl is extremely low the use of neuroimaging screening may no longer be warranted.

\section{Authors' affiliations}

M Ruggieri, Institute of Neurological Science, National Research Council (CNR), Catania, Italy

M Ruggieri, P Pavone, A Polizzi, Department of Paediatrics, University of Catania, Italy

M Di Pietro, A Scuderi, Paediatric Ophthalmology Unit, Department of Paediatrics, University of Catania, Italy

A Gabriele, Molecular Genetic Unit, Institute of Neurological Science, National Research Council (CNR), Cosenza, Italy

A Spalice, P lannetti, 2nd Chair of Paediatrics, Division of Paediatric Neurology, Department of Paediatrics, University "La Sapienza," Rome, Italy

\section{REFERENCES}

1 Happle RH. A rule concerning the segmental manifestation of autosomal dominant skin disorders. Review of clinical examples providing evidence for dichotomous types of severity. Arch Dermatol 1997;133:1505-9.

2 Zlotogora J. Germ line mosaicism. Hum Genet 1998;102:381-6.

3 Itin PH, Buechner SA. Segmental forms of autosomal dominant skin disorders: the puzzle of mosaicism. Am J Med Genet 1999:86:351-4.

4 Rott HD. Extracutaneous anomalies of Blaschko lines. Am J Med Genet 1999:85:338-41.

5 Moss C, Green SH. What is segmental neurofibromatosis ? Br J Dermatol 1994;130:106-10.

6 Huson SM, Ruggieri M. The neurofibromatoses. In: Harper J, Oranie JM Rose M, eds. Textbook of pediatric dermatology.Vol II. Oxford: Blackwell Science, 2000: 1204-24.

7 Mac Collin M, Woodfin W, Kronn D, et al. Schwannomatosis: a clinical and pathologic study. Neurology 1996;46:1072-9.

8 Antinheimo J, Sankila R, Carpèn O, et al. Population-based analysis of sporadic and type 2 neurofibromatosis-associated meningiomas and Schwannomas. Neurology 2000;54:71-6.

9 Ingordo V, D'Andria G, Mendicini S, et al. Segmental neurofibromatosis: is it uncommon or underdiagnosed? Arch Dermatol 1995;131:959-60.

10 Wolkenstein P, Mahmoudi A, Zeller J, et al. More on the frequency of segmental neurofibromatosis. Arch Dermatol 1995;131:1465.

11 Hager CM, Cohen PR, Tschen JA. Segmental neurofibromatosis: case report and review. J Am Acad Dermatol 1997;37:864-9.

12 Wallace $M$, Baver $M$, Rasmussen $S$, et al. Segmental and mosaic forms of neurofibromatosis 1: molecular and embryological insights. Am J Hum Genet. 1997;61: A412 (Abstract)).

13 Hix K, Rothner D, Cohen B. Segmental neurofibromatosis: analysis of 14 cases. Ann Neurol. 1998;44: 575-6 (Abstract))

14 Ruggieri M, Huson SM. The clinical and diagnostic implications of mosaicism in the neurofibromatoses. Neurology 2001;56:1434-43.

15 Gutman DH, Aynsworth A, Carey JC, et al. The diagnostic evaluation and multidisciplinary management of neurofibromatosis 1 and neurofibromatosis 2. JAMA 1997;278:51-7. 
16 Friedman J, Gutman DH, MacCollin M, et al. In: Neurofibromatosis: phenotype, natural history and pathogenesis, 3rd ed. Baltimore: Johns Hopkins University Press, 1999.

17 Tinschert S, Naumann I, Stegmann E, et al. Segmental neurofibromatosis is caused by somatic mutation of the neurofibromatosis type 1 (NF1) gene. Eur J Hum Genet 2000;8:455-9.

18 Vandenbroucke I, Van Doorn R, Callens T, et al. Genetic and clinical mosaicism in a patient with neurofibromatosis type 1. Hum Genet 2004; 114:284-90

19 MacCollin $M$, Jacoby LB, Jones $D$, et al. Somatic mosaicism of the neurofibromatosis type 2 suppressor-gene. Neurology 1997;48:A429.

20 Viskochil D, Carey JC. Alternate forms of NF1 and NF2. In: Huson SM Hughes RAC, eds. The neurofibromatoses: a clinical and pathogenetic overview. London: Chapman and Hall, 1994:455-74.

21 NIH Consensus Development Conference Statement. Neurofibromatosis. Arch Neurol 1988;45:575-8.

22 Wolkenstein $\mathrm{P}$, Freche B, Zeller J, et al. Usefulness of screening investigations in neurofibromatosis type 1. A study of 152 patients. Arch Dermatol 1996;132:1333-6.

23 Ruggieri M. The different forms of neurofibromatosis. Child's Nerv Syst 1999; 15:295-308.

24 Ruggieri M. Mosaic (segmental) neurofibromatosis type 1 (NF1) and type 2 (NF2): no longer neurofibromatosis type 5 (NF5). Am J Med Genet $2001 ; 101: 178-80$

25 Riccardi VM, Lewis RA. Penetrance of von Recklingausen neurofibromatosis: a distinction between predecessors and descendants. Am J Hum Genet 1988;42:284-9.

26 Huson SM, Hughes RAC. The neurofibromatoses: pathogenic and clinical overview. London: Chapman and Hall, 1994:253-74.

27 Beauchamp G. Neurofibromatosis type 1 in children. Trans Am Ophthal Soc 1995;93:445-72.
28 Listernick R, Mancini AJ Charrow J. Segmental neurofibromatosis in childhood. Am J Med Genet 2003;121A:132-5.

29 Blakley P, Louis DN, Short MP, et al. A clinical study of patients with multiple isolated neurofibromas. J Med Genet 2001;38:485-8.

30 MacCollin M, Willett C, Heinrich B, et al. Familial schwannomatosis: exclusion of the NF2 locus as the germline event. Neurology 2003;60:1968-74.

31 Leverkus M, Kluwe L, Roll EM, et al. Multiple unilateral schwannomas: segmental neurofibromatosis type 2 or Schwannomatosis? Br J Dermatol 2003; 148:804-9

32 Brudler $\mathrm{CE}$, Ichimura $\mathrm{K}$, Tingby $\mathrm{O}$, et al. A group of schwannomas with interstitial deletions on 22 located outside the NF2 locus show no detectable mutations in the NF2 gene. Hum Genet 1999;104:418-24.

33 Kauffman DL, Heinrich BS, Willett C, et al. Somatic instability of the NF2 gene in schwannomatosis. Arch Neurol 2003;60:1317-20.

34 Riccardi VM. Neurofibromatosis: clinical heterogeneity. Curr Prob/ Cancer 1982;7:1-34

35 Rubenstein A, Bader JL, Aron AA, et al. Familial transmission of segmental neurofibromatosis. Neurology. 1983;33: 76 (Abstract), (S2).

36 Boltshauser E, Stocker H, Machler M. Neurofibromatosis type 1 in a child of a parent with segmental neurofibromatosis (NF-5). Neurofibromatosis $1989 ; 2: 244-5$

37 Uhlin SR. Segmental neurofibromatosis. South Med J 1980;73:526-7.

38 Segal R. Segmental neurofibromatosis of the sciatic nerve: case report. Neurosurgery 1993;33:948.

39 Lazaro C, Ravella A, Gaona A, et al. Neurofibromatosis type 1 due to germ-line mosaicism in a clinically normal father. $N$ Engl J Med 1994;331:1403-7.

40 Gardner RL. Cell lineage and cell commitment in the early mammalian embryo. In: Warshaw JB, eds. The biological basis of reproductive and developmental medicine. Amsterdm: Elsevier, 1983:31-41. 